\title{
Nonsingular Chaplygin gas cosmologies in universes connected by wormhole
}

\author{
Anna Mokeeva ${ }^{1) \dagger}$ and Vladimir Popov ${ }^{2) \ddagger}$ \\ 1) Physical Department, Lobachevsky State University of Nizhni Novgorod, Gagarin ave. 23, Nizhni \\ Novgorod 603950, Russia \\ ${ }^{2)}$ Institute of Physics, Kazan Federal University, Kremlyovskaya st. 18, Kazan 420008, Russia \\ †anne.zaharovoy@gmail.com \\ ‡vladipopov@mail.ru
}

\begin{abstract}
We present some exact solutions of the Einstein equations with an anisotropic fluid exploiting the Chaplygin equation of state. The solutions describe spacetimes with two identical $\mathrm{T}$ regions and an intermediate static spherically symmetric $\mathrm{R}$ region containing a wormhole. The metric in the $\mathrm{T}$ region represents an anisotropic Kantowski-Sachs cosmological model. Its evolution starts from a horizon and develops according to different scenarios including eternal expansion, contraction and also a finite universe lifetime.
\end{abstract}

PACS: 04.20.-q, 04.20.Jb, 98.80.Bp 


\section{Introduction}

Observational data of the last decade, such as Type Ia Supernovae (SNIa) [1], Cosmic Microwave Background (CMB) [2] and Large Scale Structure [3], are evidence of accelerating expansion of the Universe. This behavior is caused by the dark energy featured by a negative ratio of the pressure to the energy density. A dark energy nature is not clear nowadays and a number of competing models describe the dark energy as an exotic matter or as a result of non-minimal gravitational interaction with ordinary matter.

The simplest dark energy model, the cosmological constant, is indeed the vacuum energy with the equation of state $p=-\rho$. A number of models, such as quintessence [4], k-essence [5], phantom [6] and etc., are based on scalar field theories. An alternative way is to be thought a geometric origin of the dark energy. It motivates to modify the four-dimensional gravity theory [7] or to introduce additional dimensions as in braneworld models [8]. The Chaplygin gas model, also denoted as quartessence, exploits a negative pressure fluid, which is inversely proportional to the energy density $p=-\alpha / \rho[9]$. It was modified for the models unifying the dark energy and dark matter [10], including generalized Chaplygin gas [11, 12] and superfluid Chaplygin gas [13]. For a more detail review of dark energy models and references see [14].

In the cosmological context the Chaplygin gas originally appeared as a hydrodynamical representation of the generalized Born-Infeld Lagrangian

$$
\mathcal{L}_{\mathrm{BI}}=-\sqrt{\alpha} \sqrt{1-\partial_{\nu} \theta \partial^{\nu} \theta}
$$

describing a (3+1)-dimensional brane universe with the scalar field $\theta$ in a (4+1)-dimensional bulk [11]. It was also shown that the Chaplygin gas model can be derived from the Lagrangian

$$
\mathcal{L}=\partial_{\nu} \phi^{*} \partial^{\nu} \phi-M\left(\frac{\phi^{*} \phi}{\lambda}+\frac{\lambda}{\phi^{*} \phi}\right)
$$

for a complex scalar field $\phi$ in the WKB-approximation [13].

The Chaplygin equation of state can violate the strong energy condition $\rho+3 p>0$, which is necessary for an accelerating cosmological process as well as it can violate the null energy condition $\rho+p>0$ providing for the existence of traversable wormholes. Wormholes with the Chaplygin gas are originally studied by Lobo [15]. Owing to the peculiar equation of state the obtained solutions possess some features. Primarily, if the solution exists in the whole spacetime then it cannot be asymptotically flat. To ensure asymptotic flatness for the Chaplygin wormhole the interior solution in [15] is matched with the exterior Schwarzschild solution using the Darmois-Israel formalism of a dynamic thin shell [16]. This method is also used when the wormhole solution exists only within a spatial sphere and cannot be directly continued outside because it is singular on the sphere.

An alternative approach to the Chaplygin wormholes is to consider horizons instead of singularities. A cosmic expansion beyond an event horizon can be considered as a model of an origin of our Universe $[17,18,19,20]$. The idea that the universe can emerge in the interior of a 
black hole was proposed by Pathria [17]. The primary attention to the universes inside the black holes is concentrated on the inquiry of geometrical properties and cosmological details rather than on exact solutions of the Einstein equations [18]. Bronnikov et al. [19] used the exact solutions for phantom scalar fields to study and classify regular black holes where singularity is replaced by a cosmological expansion and which therefore were called black universes. Such objects can be supported by phantom matters and could arise from collapse in another ambient universe. In the work [20] the Lemaître type cosmology for an anisotropic perfect fluid with the vacuum equation of state was considered for different topologies of the spacetime. The conditions for the cosmological evolution starting from a horizon for different kinds of matter were studied in [21, 22].

We apply the similar structure to Chaplygin wormholes. It is free of singularities and the wormhole is surrounded by horizons separating the spacetime into the static and cosmological regions. In this work we deal with the simple horizons for which we determine the conditions of regularity for the metric and the Chaplygin matter. Some exact solutions corresponding to different cosmological scenarios are developed using these conditions.

The paper is organized as follows. In the next section we consider the general properties of spacetimes with a spherically symmetric wormhole surrounded by two horizons and external universes which are supported by matter with the Chaplygin equation of state. Some particular cosmological scenarios are presented in Sec. 3. Concluding remarks are summarized in Sec. 4.

\section{Wormhole between two horizons}

We consider a static spherically symmetric spacetime with the metric

$$
\mathrm{d} s^{2}=A(r) \mathrm{d} t^{2}-\frac{\mathrm{d} r^{2}}{1-b(r) / r}-r^{2}\left(\mathrm{~d} \theta^{2}+\sin ^{2} \theta \mathrm{d} \varphi^{2}\right)
$$

and the stress-energy tensor of an anisotropic perfect fluid in which the energy density and the radial pressure are related by Chaplygin's equation of state

$$
p_{r}=-\frac{\alpha}{\rho}
$$

where $\alpha$ is a positive constant. In this case the Einstein equations $G_{\mu \nu}=8 \pi T_{\mu \nu}$ (we set $c=G=1$ ) reduce to

$$
\begin{aligned}
b^{\prime} & =8 \pi r^{2} \rho \\
\frac{A^{\prime}}{A} & =\frac{b+8 \pi r^{3} p_{r}}{r^{2}(1-b / r)} \\
p_{r}^{\prime} & =\frac{2}{r}\left(p_{t}-p_{r}\right)-\left(\rho+p_{r}\right) \frac{A^{\prime}}{A}
\end{aligned}
$$

where the prime denotes the derivative with respect to $r$ and $p_{t}(r)$ is the transversal pressure. 
The metric (3) describes a static spherically symmetric wormhole if the radial coordinate $r$ increases from a minimum value, corresponding to the wormhole throat, to infinity. Without loss in generality we take $r_{\min }=1$. In this approach $r$ is associated with two maps covering the spacetime on either side of the throat. The functions $A(r)$ and $b(r)$ are regular and defined through all the range $1 \leq r<\infty$ and satisfy the flare-out conditions

$$
\begin{gathered}
b(1)=1, \quad b^{\prime}(1)<1 \\
0<A(1)<\infty, \quad A^{\prime}(1)<\infty
\end{gathered}
$$

to provide a minimal area for coordinate spheres at $r=1$ and the metric regularity on the throat.

For traversable wormholes it is also necessary that $A(r)$ should be positive and $b(r)<r$ elsewhere to avoid horizons and singularities. These requirements are ruled out in the present paper in favor of the horizon located at the radius $r=r_{0}>1$.

The horizon conditions correspond to simultaneous solutions of the equations $b\left(r_{0}\right)=r_{0}$ and $A\left(r_{0}\right)=0$ as it is depicted in Fig. 1. We deal with only simple horizons. It means that the metric component $g_{r r}$ has a simple pole at the point $r_{0}$, so that

$$
b(r)=r_{0}+b^{\prime}\left(r_{0}\right)\left(r-r_{0}\right)+o\left(r-r_{0}\right)
$$

and the function $A(r)$ has a simple zero

$$
A(r) \propto\left(r-r_{0}\right)+o\left(r-r_{0}\right)
$$

These horizon conditions and compatibility of the Einstein equations near the horizon lead to the following relation for the Chaplygin gas

$$
b^{\prime}\left(r_{0}\right)=8 \pi r_{0}^{2} \sqrt{\alpha}
$$

In this case $r_{0}$ corresponds to the simple horizon separating a static $\mathrm{R}$ region at $r<r_{0}$ from a non-static $\mathrm{T}$ region at $r>r_{0}$. Indeed, for the metric (3) the square of the normal to a surface $r=$ const is $\Delta=b / r-1$ and $\Delta<0$ when $1<r<r_{0}$. In this region the spacetime is static ( $\mathrm{R}$ region) and $r$ is a spatial coordinate. At $r>r_{0}$ the normal is a spacelike vector and $\Delta>0$. This is a non-static $\mathrm{T}$ region where $r$ becomes a temporal coordinate.

This class of metrics is regular in any finite region for $r \geq 1$. It is verified using the Kretschmann scalar $K=R_{\alpha \beta \gamma \delta} R^{\alpha \beta \gamma \delta}$, where $R_{\alpha \beta \gamma \delta}$ is the Riemann curvature tensor. To avoid too cumbersome representation for the Kretschmann scalar we write it in the form

$$
K(r)=\frac{P\left(r, A, A^{\prime}, A^{\prime \prime}, b, b^{\prime}\right)}{A^{4}(r) r^{6}},
$$

where the numerator is a polynomial function depending on the indicated arguments and $P \propto$ $\left(r-r_{0}\right)^{4}+o\left(\left[r-r_{0}\right]^{4}\right)$ near the horizon $r=r_{0}$. Taking into account the expansion (11) it means that the Kretschmann scalar is finite on the horizon. 


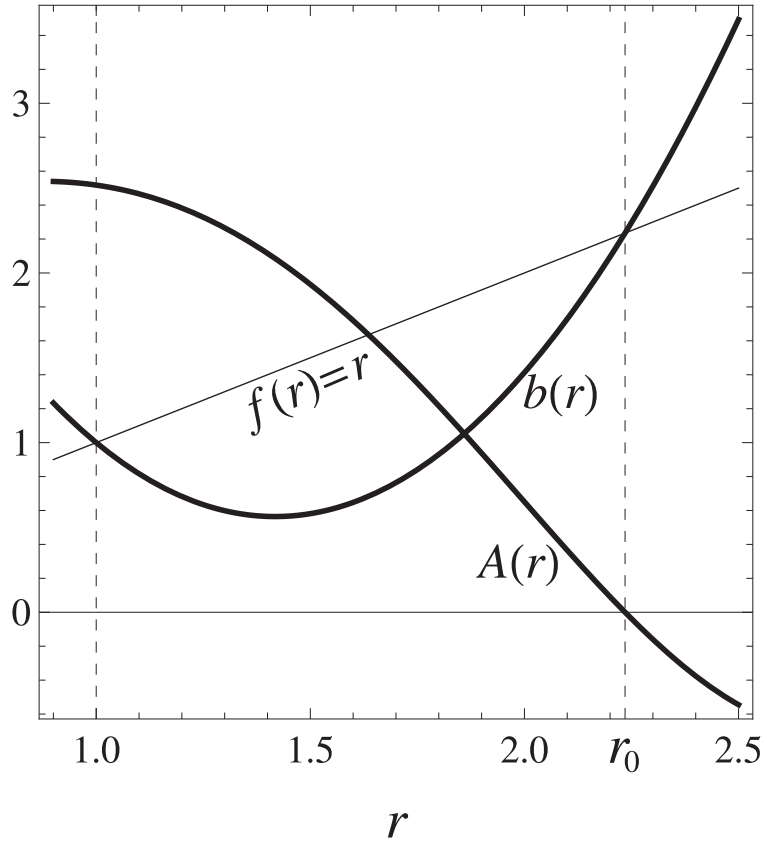

Figure 1: The functions $A(r)$ and $b(r)$ for the geometry with a wormhole at $r=1$ and simple horizons at $r=r_{0}$.

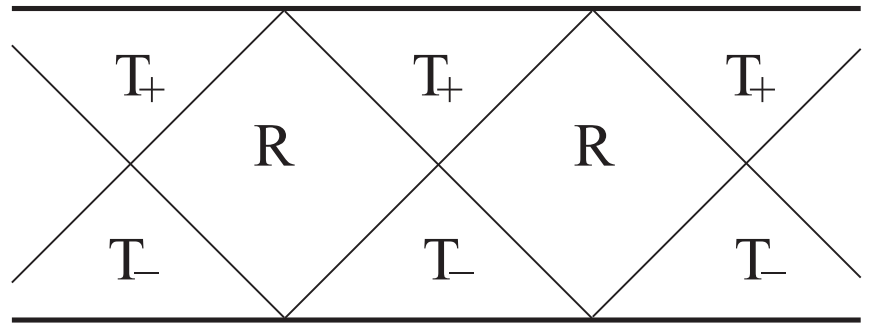

Figure 2: The global structure diagram of the model. The thick lines correspond to $r=\infty$ and the thin lines correspond to the horizons at $r=r_{0}$.

The metric (3) in the $\mathrm{T}$ region can be rewritten as

$$
\mathrm{d} s^{2}=\frac{\mathrm{d} \eta^{2}}{b(\eta) / \eta-1}-\tilde{A}(\eta) \mathrm{d} \rho^{2}-\eta^{2} \mathrm{~d} \Omega^{2},
$$

where $\tilde{A}(r)=-A(r)$ is the positive function when $r>r_{0}$. We introduce the new coordinates, $\eta$ instead $r$ and $\rho$ instead $t$ to mark the interchange of roles between the spatial and temporal coordinates. The integral

$$
\tau= \pm \int \frac{\mathrm{d} \eta}{\sqrt{b(\eta) / \eta-1}}
$$

defines the physical time $\tau$ for an observer in the $\mathrm{T}$ region. The metric (14) describes a Kantowski-Sachs (KS) anisotropic cosmological model [23] with two scale factors for the spherical and longitudinal directions which depend on the proper time $\tau$ according to $a_{t}(\tau)=\eta(\tau)$ and $a_{r}(\tau)=\tilde{A}^{1 / 2}(\eta(\tau))$ correspondingly. The global structure of the spacetime is shown in Fig. 2. The metric (14) describes both the contracting region $\mathrm{T}_{-}$corresponding to $\tau<0$ and the expanding region $\mathrm{T}_{+}$corresponding to $\tau>0$.

At $\eta=r_{0}$ there is a coordinate singularity which the observer interprets as the beginning of the universe. In [20] it is called a null bang sinse a horizon is a null surface where the spatial volume vanishes. It is easy to see from Eq. (15) that if the function $b \rightarrow \infty$ faster than $\eta^{3}$ for $\eta \rightarrow \infty$, then the universe evolves during a finite cosmological time and otherwise it lives endlessly. 
It is evident from the Einstein equations that the following conditions are fulfilled on the horizon

$$
\rho\left(r_{0}\right)=-p_{r}\left(r_{0}\right)=\sqrt{\alpha}
$$

that corresponds to the requirement $p_{r}+\rho=0$ on the horizon [21] which is independent of the matter kind. The condition (16) provides continuity of the energy density and the radial pressure in going from $\mathrm{R}$ to $\mathrm{T}$ region. In fact, $\rho$ and $p_{r}$ interchange their positions in the stressenergy tensor when crossing the horizon. The equation of state (4) is precisely the same in both regions. Under the conditions (11) and (12) the transversal pressure is also a continuous function and takes the value $p_{t}\left(r_{0}\right)=b^{\prime \prime}\left(r_{0}\right) / 8 \pi r_{0}-3 \sqrt{\alpha}$ on the horizon.

Below some exact solutions of the equations (4)-(7) with the conditions (8)-(12) corresponding to different cosmological scenarios are studied.

\section{Different types of Kantowski-Sachs cosmologies}

The equation of state (4) relates the energy density with the radial pressure only. It allows one to solve the problem predetermining the metric function $b(r)$ satisfying the flare-out and horizon conditions. The other functions are found from Eqs. (5)-(7) and produce different cosmological scenarios in the $\mathrm{T}$ regions whereas the $\mathrm{R}$ regions are similar in all solutions.

\subsection{Eternally expanding universes}

One of the simplest functions satisfying the conditions (8) and (10) is the parabola

$$
b(r)=d(r-1)\left(r-r_{0}\right)+r
$$

The function $A(r)$ is expressed from Eq. (6) with the conditions (9) and (11) as

$$
A(r)=A_{0} \frac{\left(r_{0}-r\right)\left(r+q_{1}\right)^{q_{2}}}{r} e^{q_{3} r+q_{4} r^{2}}
$$

where

$$
\begin{gathered}
q_{1}=\frac{r_{0} d+d-1}{2 d}, \quad q_{2}=\frac{2 q_{1}^{4}}{r_{0}^{4}} \frac{1-d+r_{0} d}{1+d-r_{0} d}, \quad q_{3}=2 q_{4}\left(3 q_{1}+\frac{1}{d}\right), \\
q_{4}=\frac{\left(r_{0} d-d+1\right)^{2}}{4 r_{0}^{4} d^{2}}, \quad d=\frac{r_{0}^{2} \sqrt{r_{0}^{4}+8}-r_{0}^{4}-2}{2\left(r_{0}-1\right)}
\end{gathered}
$$

are positive constants and $r_{0}$ is connected with the parameter $\alpha$ from the equation of state (4) by the relation

$$
\alpha=\frac{\left(1-d-r_{0} d\right)^{2}}{64 \pi^{2} r_{0}^{4}} .
$$

In the $\mathrm{R}$ region the metric (3) can be transformed to the form

$$
\mathrm{d} s^{2}=B(x) \mathrm{d} t^{2}-\mathrm{d} x^{2}-r^{2}(x)\left(\mathrm{d} \theta^{2}+\sin ^{2} \theta \mathrm{d} \varphi^{2}\right)
$$




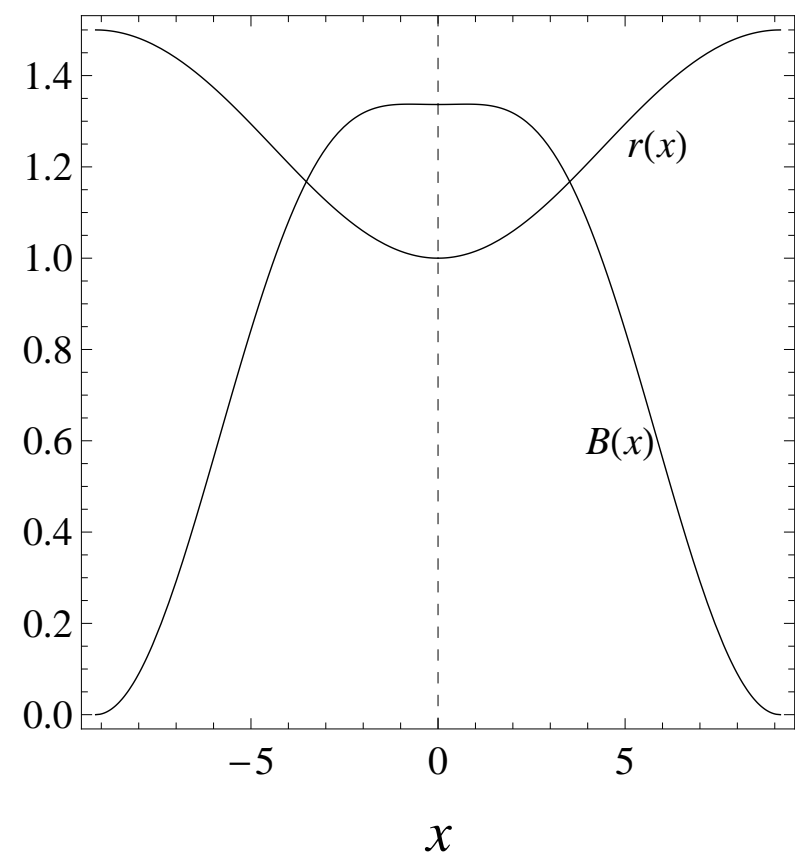

Figure 3: The metric functions depending on the Gaussian coordinates in the $\mathrm{R}$ region. This figure is plotted for $b(r)$ given by Eq. (17) when $r_{0}=1.5$ and other parameters are calculated according to (19), however the functions $r(x)$ and $B(x)$ have a similar form for the other solutions.

where

$$
r(x)=1+\left(r_{0}-1\right) \sin ^{2} \varepsilon(\sqrt{d} x / 2),
$$

and $\varepsilon(z)$ is the inverse function to the elliptic integral of the second kind $E\left(\varepsilon \mid 1-r_{0}\right)$. The metric function $B(x)=A(r(x)$ ), where $r(x)$ from Eq. (22) is substituted into the expression (18). Behaviors of $r(x)$ and $B(x)$ are depicted in Fig. 3.

Contrary to the representation (3), the metric (21) describes the whole $\mathrm{R}$ region with the Gaussian radial coordinate $x$ lying in the range $|x| \leq \pi E\left(\pi / 2 \mid 1-r_{0}\right) / \sqrt{d}$. When $x \rightarrow$ $\pm \pi E\left(\pi / 2 \mid 1-r_{0}\right) / \sqrt{d}$ the function $B(x)$ becomes zero providing the evidence for horizons which are spaced at a finite distance apart for a static observer. The function $r(x)$ has a minimum at $x=0$ implying a wormhole throat, so the $\mathrm{R}$ region is a spherically symmetric one with the wormhole surrounded by two horizons.

For observers in the $\mathrm{T}$ region the universe evolution starts from the horizon at some instant in the past and lasts for infinite time. All this time the universe expands in all directions as it demonstrates in Fig. 4. In the far future the scale factor along the coordinate spheres is quadratic in the proper time $\tau$ while the scale factor in the radial direction grows exponentially

$$
a_{r} \propto \tau^{q_{2}} \exp q_{4} \tau^{4} / 2, \quad a_{t} \propto \tau^{2} .
$$




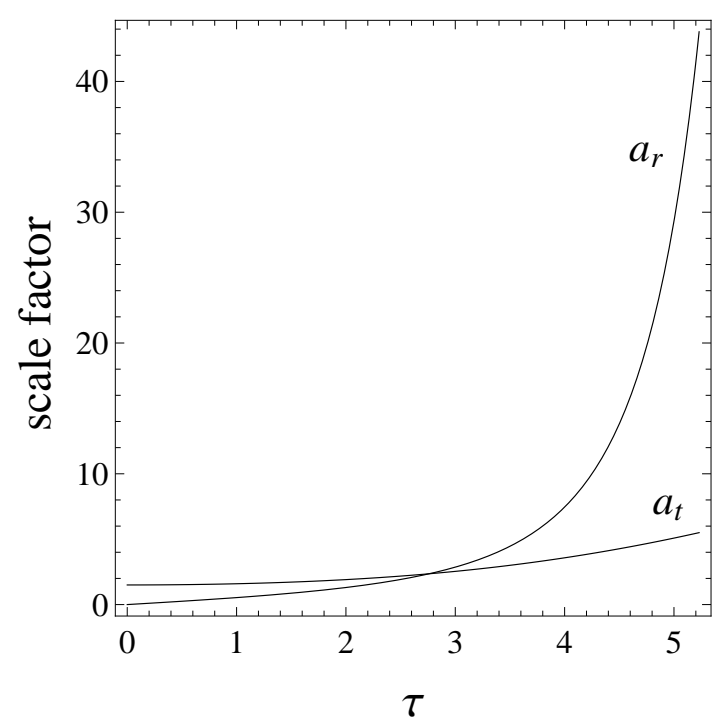

Figure 4: The increase of the scale factors for the solution specified by Eqs. (17)-(19) for $r_{0}=1.5$.

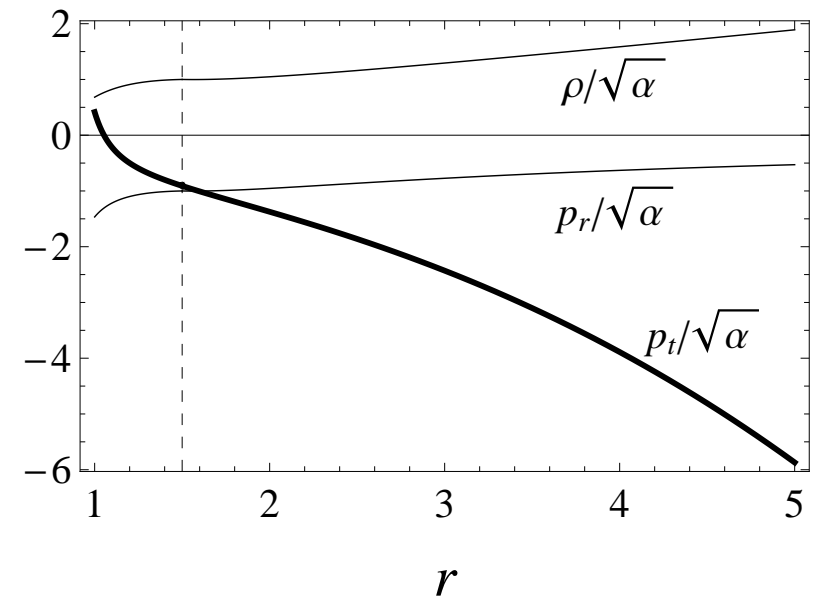

Figure 5: The dimensionless energy density, radial and transversal pressures as functions of the radial coordinate for the eternally expanding universe.

The behaviors of the energy density and the pressures are shown in Fig. 5. The energy density is positive and the radial pressure is negative in the whole spacetime whereas the transversal pressure reverses its sign. In the $\mathrm{T}$ region all these quantities are comparable close to the null bang. At the late stage the radial pressure decreases in its absolute value as $p_{r} \propto \tau^{-2}$ and the main contribution is given by the energy density growing quadratically in the proper time, $\rho \propto \tau^{2}$, and the negative transversal pressure which increases in its absolute value as $p_{t} \propto \tau^{6}$.

\subsection{Universes with finite lifetime}

The alternative scenario is arrived from the equations (4)-(7) if the function

$$
b(r)=d r^{4}+1-d .
$$

In this case the function

$$
A(r)=A_{0} \frac{\left(r_{0}-r\right)}{r\left[r^{2}+\left(r_{0}+1\right) r+q_{1}^{2} q_{2}+r_{0}\right]^{q_{1}}} \exp \left\{-\frac{4 r_{0} q_{1}}{q_{2}} \arctan \left(\frac{2 r+r_{0}+1}{q_{2}}\right)\right\}
$$

where

$$
q_{1}=\frac{r_{0}^{2}+1}{q^{2}}, \quad q_{2}=\sqrt{3 r_{0}^{2}+2 r_{0}+3}, \quad r_{0}=1+\sqrt{2}
$$

This solution implies $\alpha=(3-2 \sqrt{2}) / 64 \pi^{2}$ in the equation of state (4).

In the $\mathrm{R}$ region the spacetime has the same structure as before. The metric can be transformed to the form (21) with the only difference that the metric functions $B(x)$ and $r(x)$ cannot 


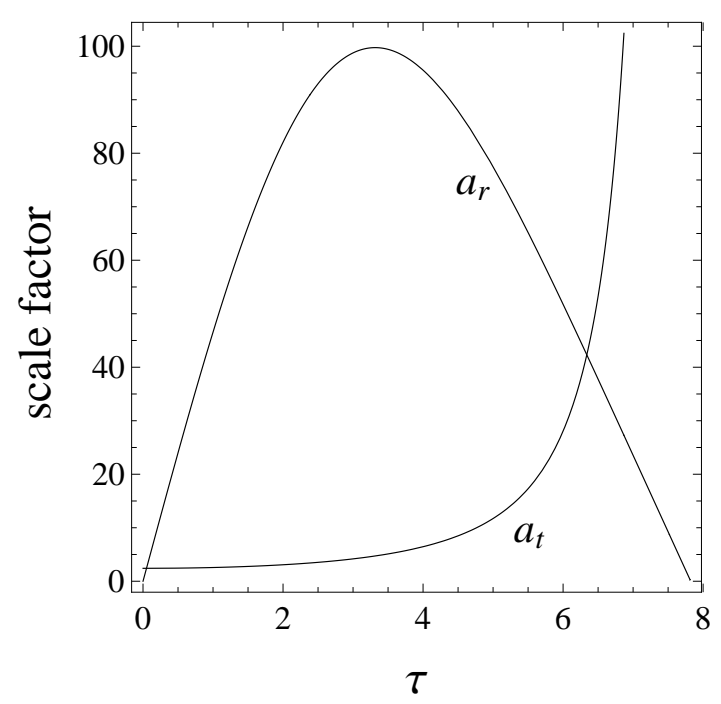

Figure 6: The behaviors of the scale factors for the solution when the function $b(r)$ and $A(r)$ are given by Eqs. (24) and (25). The proper lifetime of the universe is finite.

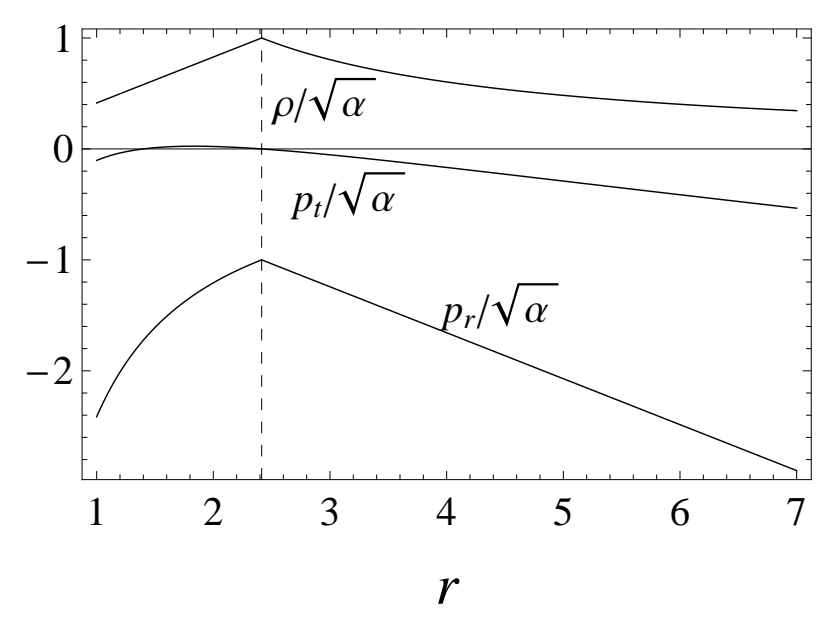

Figure 7: The energy density, radial and transversal pressures as a functions of the radial coordinate in the universe with the finite proper lifetime.

be obtained analytically and all calculations are numerical. Their qualitative behaviors are the same as in Fig. 3

The expansion in the T region starts with a null bang and the ensuing cosmological evolution develops during finite physical lifetime from the point of view of the KS observer. Fig 6 demonstrates that throughout this period the spherical scale factor infinitely increases and the longitudinal scale factor peaks at some instant and falls to zero, although both radial and transversal pressures are negative and the energy density disappears towards the end of the evolution as it is shown in Fig. 7. This situation arises in the KS model from the fact that a transversal acceleration is determined by the radial pressure and the transversal Habble parameter $H_{t}=\dot{a}_{t} / a_{t}$, where the dot denotes the derivative with respect to the proper time $\tau$, while a radial acceleration also depends on the transversal pressure and the energy density according to

$$
\begin{gathered}
\frac{1}{a_{t}^{2}}+H_{t}^{2}+2 \frac{\ddot{a}_{t}}{a_{t}}=-8 \pi p_{r} \\
\frac{1}{a_{t}^{2}}+H_{t}^{2}-\frac{\ddot{a}_{r}}{a_{r}}=4 \pi\left(\rho-p_{r}+2 p_{t}\right) .
\end{gathered}
$$

If the energy density is neglected and the rate of growth of $H_{t}$ is small so that $\left|p_{r}\right|>2\left|p_{t}\right|+$ $H_{t}^{2} / 4 \pi$, then one observes a decelerated expansion or contraction in the longitudinal direction. 


\subsection{Eternally contracting universes}

An intermediate scenario is obtained for the metric functions

$$
\begin{gathered}
b(r)=d r(r-1)\left(r-r_{0}\right)+r, \\
A(r)=A_{0} \frac{\left(r_{0}-r\right)}{r^{q_{3}}\left[3 r^{2}-2 q_{1} r+r_{0} q_{3}\right]^{q_{2} q_{5}}} \exp \left\{-2 \frac{q_{1} q_{4} q_{5}}{q_{6}} \arctan \left(\frac{3 r-q_{1}}{q_{6}}\right)\right\},
\end{gathered}
$$

where

$$
\begin{gathered}
q_{1}=r_{0}+1, \quad q_{2}=d\left(2+5 r_{0}+2 r_{0}^{2}\right)-2 d^{2} r_{0}\left(1+r_{0}+r_{0}^{2}\right)-1, \\
q_{3}=1+\frac{1}{d r_{0}}, \quad q_{4}=d\left(2+5 r_{0}+2 r_{0}^{2}\right)-d^{2} r_{0}\left(2-r_{0}+2 r_{0}^{2}\right)-4, \\
q_{5}=\frac{1-d r_{0}+d r_{0}^{2}}{6 d^{2} r_{0}^{4}\left(1+d-d r_{0}\right)}, \quad q_{6}=\sqrt{\frac{3}{d}-1+r_{0}-r_{0}^{2}}, \\
\alpha=\frac{\left(1-d r_{0}+d r_{0}^{2}\right)^{2}}{64 \pi^{2} r_{0}^{2}}, \quad d=\frac{r_{0} \sqrt{r_{0}^{4}+4 r_{0}^{2}+4 r_{0}}-r_{0}^{3}-2}{2\left(r_{0}^{2}-1\right)}
\end{gathered}
$$

are positive constants except $q_{2}$, which can be both positive and negative ${ }^{1}$, and the horizon radius lies in the range $1<r_{0}<r_{\max } \approx 3.04$. If $r_{0} \geq r_{\max }$ the function $A(r)$ resulting from Eq. (6) gets singularities in the static region.

Note that the transformation to the metric (21) in the R region gives the quite simple form for spherical radii

$$
r(x)=\frac{1}{2}\left(\left(r_{0}+1\right)-\left(r_{0}-1\right) \cos \sqrt{d} x\right)
$$

with the Gaussian coordinate $x$ lying in the range $[-\pi / \sqrt{d}, \pi / \sqrt{d}]$.

For the KS observer the universe starts to evolve from the horizon and gradually dies into infinite future because the expansion in the radial direction gives place to the contraction. The scale factors for the longitudinal and lateral directions as functions of the proper time are shown in Fig. 8. The radial pressure is everywhere negative (see Fig. 9), so that it guarantees the accelerated expansion along the coordinate spheres, and the inequality $\rho-p_{r}+2 p_{t}-H_{t}^{2} / 4 \pi>0$ provides the decelerated growth and the subsequent decay of the radial scale factor according to Eq. (28). At the late stage the scale factors vary in the proper time $\tau$ by the exponential laws

$$
a_{r} \propto \exp \left\{-\sqrt{d}\left(q_{2} q_{5}-\frac{1}{2 d r_{0}}\right) \tau\right\}, \quad a_{t} \propto \exp \sqrt{d} \tau .
$$

The asymptotic expression (33) resembles in some way the de Sitter behavior bringing the pressures and the energy density to the constant values

$$
\rho \rightarrow \frac{8 \pi \alpha}{3 d}, \quad p_{r} \rightarrow-\frac{3 d}{8 \pi}, \quad p_{t} \rightarrow-\frac{d}{32 \pi}\left[4+\left(3-q_{1}-2 q_{2} q_{3}\right)\left(1-q_{1}-2 q_{2} q_{3}\right)\right] .
$$

Such a tendency takes place for any function $b(\eta)$ increasing as $\eta^{3}$ when $\eta \rightarrow \infty$. It can provide the basis for a cosmological scenario with an asymptotic de Sitter phase at late times.

\footnotetext{
${ }^{1}$ Nevertheless the value $q_{3}+2 q_{2} q_{5}-1>0$ for all values of $r$ lying in the range $1<r_{0}<r_{\max }$. It means that $A(r)$ vanishes when $r \rightarrow \infty$.
} 


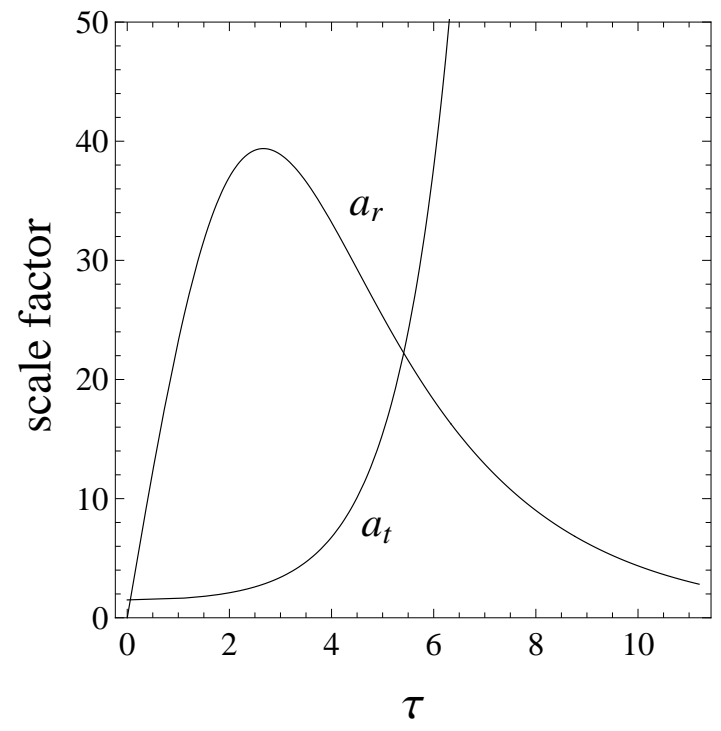

Figure 8: The behaviors of the scale factors in the solution with the metric function (29) and (30) where the quantities (31) are calculated for $r_{0}=1.5$. The universe expands in the spherical direction and contracts in the radial direction during an infinite time.

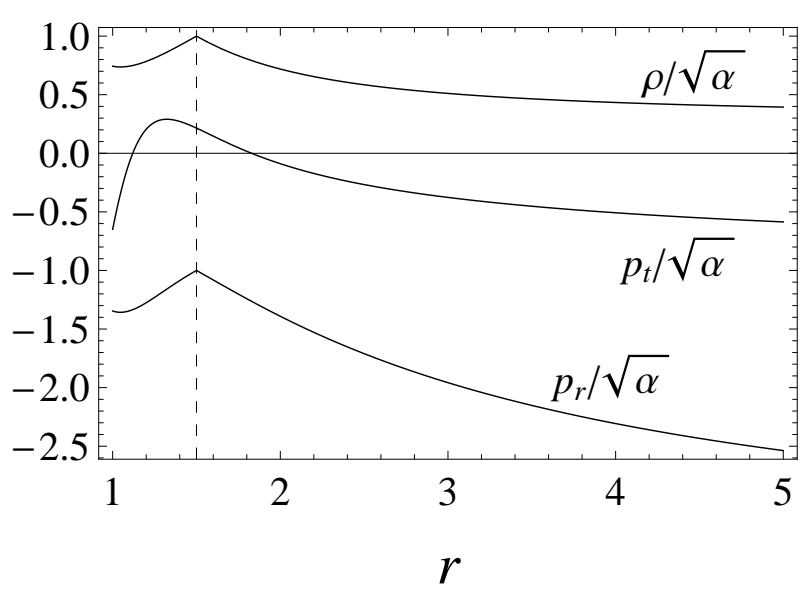

Figure 9: The energy density, radial and transversal pressures as functions of the radial coordinate in the gradually dying universe.

\section{Conclusion}

We have considered some exact solutions of Einstein's equations with anisotropic fluid describing a spacetime with two identical cosmological T regions and an intermediate static spherically symmetric $\mathrm{R}$ region. The latter contains a wormhole allowing one to pass between two horizons. The T regions are described by anisotropic KS models with different kinds of cosmological evolution. We have presented the solutions describing eternally expanding universes, disappearing ones and universes with a limited lifetime.

These solutions rest upon the flare-out conditions (8), (9) and the horizon regularity conditions for matter with the Chaplygin equation of state (10)-(12), (16). Similar regularity conditions were derived before for the null bang cosmologies with different types of matters such as the neutral and charged matter with a barotropic equation of state $p_{r} \sim \rho^{m}, m \geq 1$ [22] and matter with a non-vacuum behavior (i.e. the ratio of the radial pressure to the energy density $w=p_{r} / \rho \neq-1$ everywhere) [21]. However they are incompatible with the model under consideration since the regularity for the Chaplygin gas is realized only through the "vacuum" value $w=-1$ on the horizon.

The presented solutions violate everywhere the dominated energy condition, $\rho>0, \rho>$ $\left|p_{r}\right|, \quad \rho>\left|p_{t}\right|$ while the strong energy condition proves to be realized in some parts of the 
spacetime as it takes place in the last scenario. One sees from Fig. 9 that the relation $\rho+p_{r}+$ $2 p_{t}>0$ holds in the layer near the horizon.

Note that the suggested spacetime conformation does not support an isotropic solutions in both $\mathrm{R}$ and $\mathrm{T}$ regions. Indeed, the isotropy of a spacetime implies $p_{r}=p_{t}$ and Eq. (7) is readily integrated. The resulting function $A(r)$ vanishes concurrently with the energy density and hence with the derivative $b^{\prime}(r)$. Fig. 1 pictorially shows that such a solution is incompatible with the considered structure.

Besides, there is no an isotropization in the solutions obtained, although it has considerable utility for the early Universe model. Nevertheless, isotropization can be most likely realized in the studied model. The last scenario has the asymptotically constant pressures and energy density, and solutions of this kind may quite probably demonstrate the de Sitter behavior at late times for the special selection of the metric functions. This problem is the subject of our current studies and the results will be presented in our future papers. 


\section{References}

[1] A. G. Riess et al., Astron. J. 116 (1998) 1009 [astro-ph/9805201];

S. Perlmutter et al., Astrophys. J. 517 (1999) 565 [astro-ph/9812133];

J. L. Tonry et al., Astrophys. J. 594 (2003) 1 [astro-ph/0305008];

R. A. Knop et al., Astrophys. J. 598 (2003) 102 [astro-ph/0309368].

[2] S. Masi et al., Prog. Part. Nucl. Phys. 48 (2002) 243 [astro-ph/0201137];

C. L. Bennett et al., Astrophys. J. Suppl. 148 (2003) 1 [astro-ph/0302207];

D. N. Spergel et al., Astrophys. J. Suppl. 148 (2003) 175 [astro-ph/0302209];

C. J. MacTavish et al., Astrophys. J. 647 (2006) 799 [astro-ph/0507503];

E. Komatsu et al., Astrophys. J. Suppl. 192 (2011) 18 [arXiv:1001.4538].

[3] M. Tegmark et al., Phys. Rev. D 69 (2004) 103501 [astro-ph/0310723];

A. C. Pope et al., Astrophys. J. 607 (2004) 655 [astro-ph/040124];

U. Seljak et al., Phys. Rev. D 71 (2005) 103515 [astro-ph/0407372];

K. Abazajian et al., Astrophys. J. Suppl. 182 (2009) 543 [arXiv:0812.0649].

[4] C. Wetterich, Nucl. Phys. B 302 (1988) 668;

P. J. E. Peebles and B. Ratra, Astrophys. J. 325 (1988) L17.

[5] C. Armendariz-Picon, V.F. Mukhanov and P. J. Steinhardt, Phys. Rev. Lett. 85 (2000) 4438 [astro-ph/0004134].

[6] R. R. Caldwell, M. Kamionkowski and N. N. Weinberg, Phys. Rev. Lett. 91 (2003) 071301 [astro-ph/0302506].

[7] S. Nojiri and S. D. Odintsov, Phys. Rev. D 68 (2003) 123512 [hep-th/0307288];

S. Capozziello, V.F. Cardone and A. Troisi, Phys. Rev. D 71 (2005) 043503 [astro$\mathrm{ph} / 0501426]$

L. Amendola, D. Polarski and S. Tsujikawa, Phys Rev. Lett. 98 (2007) 131302 [astro$\mathrm{ph} / 0603703]$.

[8] C. Csaki, M. Graesser, L. Randall and J. Terning, Phys. Rev. D 62 (2000) 045015 [hep$\mathrm{ph} / 9911406]$

C. Deffayet, S. J. Landau, J. Raux, M. Zaldarriaga and P. Astier, Phys. Rev. D 66 (2002) 024019 [astro-ph/0201164].

[9] A. Y. Kamenshchik, U. Moschella and V. Pasquier, Phys. Lett. B 511 (2001) 265 [grqc/0103004]

[10] L. P. Chimento, R. Lazkoz, Phys. Lett. B 615 (2005) 146 [astro-ph/0411068]. 
[11] M. C. Bento, O. Bertolami and A. A. Sen, Phys. Rev. D 66 (2002) 043507 [gr-qc/0202064]; N. Bilić, G. B. Tupper and R. D. Viollier, Phys. Lett. B 535 (2002) 17 [astro-ph/0111325].

[12] M. C. Bento, O. Bertolami and A.A. Sen, Phys. Rev.D 70 (2004) 083519 [astro$\mathrm{ph} / 0407239]$.

[13] V. A. Popov, Phys. Lett. B 686 (2010) 211 [arXiv:0912.1609].

[14] E. J. Copeland, M. Sami and S. Tsujikawa, Int. J. Mod. Phys. D 15 (2006) 1753 [hepth/0603057];

J. Frieman, M. Turner and D. Huterer, Ann. Rev. Astron. Astrophys. 46 (2008) 385 [arXiv:0803.0982];

S. Capozziello and M. Francaviglia, Gen. Rel. Grav. 40 (2008) 357 [arXiv:0706.1146];

V. Faraoni and T. Sotiriou, Rev. Mod. Phys. 82 (2010) 451 [arXiv:0805.1726].

[15] F. S. N. Lobo, Phys. Rev. D 73 (2006) 064028 [gr-qc/0511003].

[16] G. Darmois, Memorial des sciences mathematiques XXV, Fascicule XXV, ch. V, GauthierVillars, Paris, France, 1927;

W. Israel, Nuovo Cimento 44B (1966) 1.

[17] R. K. Pathria, Nature 240 (1972) 298.

[18] V. P. Frolov, M. A. Markov and, V. F. Mukhanov, Phys. Lett. B 216 (1989) 272;

V.P. Frolov, M. A. Markov and V.F. Mukhanov, Phys. Rev. D 41 (1990) 383;

W. M. Stuckey, Amer. J. Phys. 62 (1994) 788;

J. Smoller and B. Temple, PNAS 100 (2003) 11216 [astro-ph/0210105];

N. J. Popławski, Phys. Lett. B 687 (2010) 110 [arXiv:0902.1994].

[19] K. A. Bronnikov and J. C. Fabris, Phys. Rev. Lett. 96 (2006) 251101 [gr-qc/0511109];

K. A. Bronnikov, H. Dehnen and V.N. Melnikov, Gen. Rel. Grav. 39 (2007) 973 [grqc/0611022].

[20] K. A. Bronnikov, A. Dobosz and I. G. Dymnikova, Class. Quant. Grav. 20 (2003) 3797 [gr-qc/0302029].

[21] K. A. Bronnikov and O. B. Zaslavskii, Class. Quant. Grav. $25 \quad$ (2008) 105015 [arXiv:0710.5618] ;

K. A. Bronnikov and O. B. Zaslavskii, Int. J. Mod. Phys. D 18 (2009) 2283 [arXiv:0905.4425]

[22] K. A. Bronnikov and O. B. Zaslavskii, Phys. Rev. D 84 (2011) 084013 [arXiv:1107.4701].

[23] R. Kantowski and R. K. Sachs, J. Math. Phys. 7 (1966) 443. 